\title{
Variation in OSBPL10 is associated with dyslipidemia
}

\author{
Hiroshi Koriyama ${ }^{1,3}$, Hironori Nakagami ${ }^{2}$, Tomohiro Katsuya ${ }^{1,3}$, Hiroshi Akasaka ${ }^{4}$, Shigeyuki Saitoh ${ }^{4}$, \\ Kazuaki Shimamoto ${ }^{4}$, Toshio Ogihara ${ }^{5}$, Yasufumi Kaneda ${ }^{2}$, Ryuichi Morishita ${ }^{3}$ and Hiromi Rakugi ${ }^{1}$
}

The oxysterol hypothesis of cholesterol homeostasis states that oxysterol mediates feedback regulation of cholesterol biosynthesis. Oxysterol-binding proteins have been implicated in the control of lipid synthesis and metabolism. In a genomewide case-control association study in Japanese individuals, we found that the three single-nucleotide polymorphisms (SNPs) with the smallest $P$-values were located in the fifth intronic region of the oxysterol-binding protein-like $10(O S B P L 10)$ gene. In this study, we examined the association between polymorphisms in the OSBPL10 gene and risk factors for the metabolic syndrome in the Tanno and Sobetsu Study. We selected four SNPs, including three non-coding SNPs in intron 5 and a coding SNP (D254N) in exon 6. Genotype frequencies of the polymorphisms satisfied the conditions for Hardy-Weinberg equilibrium. We found that the low-density lipoprotein (LDL) cholesterol of individuals with the rs2290532 (D254N) polymorphism was significantly greater in subjects with the $C C+C T$ genotype than in subjects with the TT genotype $(124.3 \pm 1.3$ vs. 111.6 \pm 4.1 $\mathrm{mg}$ per $100 \mathrm{ml}, P=0.009)$. However, there were no significant differences in systolic or diastolic blood pressure, high-density lipoprotein cholesterol or triglyceride levels. Multiple regression analysis showed that rs2290532 (D254N) was associated with LDL cholesterol independent of age, sex or body mass index. Comparison of the genotype frequency in both groups indicated that the genotype associated with low risk (TT) reduced the risk of hyper-LDL cholesterolemia significantly $(P=0.003)$, with an odds ratio of 0.35 (95\% confidence interval=0.17-0.76). Overall, the rs2290532 (D254N) polymorphism in OSBPL10 may predispose individuals with this SNP to hyper-LDL cholesterolemia.

Hypertension Research (2010) 33, 511-514; doi:10.1038/hr.2010.28; published online 12 March 2010

Keywords: dyslipidemia; LDL cholesterol; OSBPL10

\section{INTRODUCTION}

Oxysterol-binding protein (OSBP) was identified as a high-affinity cytosolic receptor for oxysterols. ${ }^{1}$ Kaudutsch proposed the 'oxysterol hypothesis' of cholesterol homeostasis, which suggests that oxysterol mediates feedback regulation of cholesterol biosynthesis rather than cholesterol regulating itself. ${ }^{2,3}$ The important players in cholesterol homeostasis are HMG-CoA reductase and low-density lipoprotein (LDL) receptor. ${ }^{4}$ Importantly, the sterol regulatory element-binding protein transcription factors were identified as regulators of the HMGCoA reductase and the LDL receptor, and exogenous oxysterols have been used to suppress the activation of sterol regulatory elementbinding proteins in cellular lipid homeostasis. ${ }^{5}$ Moreover, certain oxysterols are important ligands for LXR, which has an important function in cholesterol efflux from the cells by regulating the ATPbinding cassette proteins, $\mathrm{ABCA1}$ and ABCG1. ${ }^{6,7}$ Therefore, there is now evidence that certain endogenously produced oxysterols can serve as physiological regulators of cholesterol homeostasis.

OSBP is not limited to control of lipid synthesis and transport in cells. It has also been implicated in signal transduction, vesicular transport and lipid metabolism. ${ }^{89}$ Families of proteins with homology to the C-terminal sterol-binding domain of OSBP are present in practically all eukaryotic organisms for which sequence information is available. In human beings, the gene family consists of 12 members, including oxysterol-binding protein-like 10 (OSBPL10). Using a genome-wide association study in a Japanese population, we earlier found that single-nucleotide polymorphisms (SNPs) in the OSBPL10 gene associate susceptibility to peripheral arterial disease (PAD) (in submission). Relevant SNPs were located within intron 5 of OSBPL10. Recently, Perttila et al. ${ }^{10}$ also reported that OSBPL10 regulates cellular lipid metabolism and is associated with high triglyceride levels in Finnish subjects. In this study, we examined the association between polymorphisms in OSBPL10 and serum lipid profiles from inhabitants of Tanno and Sobetsu in rural Japan who underwent routine medical checkups.

\section{METHODS}

Study participants

We recruited 1188 subjects who underwent medical checkups in the towns of Tanno and Sobetsu in 2003. Tanno and Sobetsu are located in Hokkaido, the northernmost island of Japan. The Tanno and Sobetsu study began in 1977 with a population-based prospective cohort design. The detailed epidemiological findings have been reported elsewhere. ${ }^{11-13}$

\footnotetext{
${ }^{1}$ Department of Geriatric Medicine, Osaka University Graduate School of Medicine, Suita, Japan; ${ }^{2}$ Division of Clinical Gene Therapy, Osaka University Graduate School of Medicine, Suita, Japan; ${ }^{3}$ Division of Gene Therapy Science, Osaka University Graduate School of Medicine, Suita, Japan; ${ }^{4}$ Second Department of Internal Medicine, Sapporo Medial University School of Medicine, Sapporo, Japan and 5 Osaka General Medical Center, Osaka Prefectural Hospital Organization, Osaka, Japan Correspondence: Dr T Katsuya, Division of Clinical Gene Therapy, Osaka University Graduate School of Medicine, 2-2 Yamada-0ka, Suita 565-0871, Japan. E-mail: katsuya@cgt.med.osaka-u.ac.jp

Received 6 December 2009; revised 6 January 2010; accepted 27 January 2010; published online 12 March 2010
} 
The subjects completed a standard questionnaire regarding their medical history, alcohol consumption and tobacco use. We measured anthropometric parameters, systolic blood pressure, diastolic blood pressure, total cholesterol, triglycerides, LDL cholesterol and high-density lipoprotein (HDL) cholesterol in all subjects. Blood samples were collected in the early morning after subjects fasted for $8-11 \mathrm{~h}$. Blood pressure was measured twice with the subjects seated after $5 \mathrm{~min}$ of rest. Exclusion criteria included taking any cholesterol-lowering or anti-hypertensive medications. After excluding 95 subjects for dyslipidemia and 372 subjects for hypertension, we conducted genetic analyses on 1093 subjects for dyslipidemia and 816 subjects for hypertension.

To identify any associations between genotype and dyslipidemia, the samples (1188 subjects) were divided into groups of patients with dyslipidemia or normal cholesterol according to the Japanese criteria for dyslipidemia as defined by the Japan Atherosclerosis Society. These criteria included LDL cholesterol $>140 \mathrm{mg}$ per $100 \mathrm{ml}$, HDL cholesterol $<40 \mathrm{mg}$ per $100 \mathrm{ml}$, or triglycerides $>150 \mathrm{mg}$ per $100 \mathrm{ml}$. Subjects who took cholesterol-lowering medications were also diagnosed with dyslipidemia (95 subjects).

All subjects gave written informed consent to participate in the genetic analysis and all other procedures associated with the study. The Institutional Review Board of Osaka University and the Institutional Review Board of Sapporo Medical University both approved the study protocol.

\section{Genotyping}

Genomic DNA was extracted from $200 \mu \mathrm{l}$ of buffy coat using a QIAamp DNA Blood kit (QIAGEN K.K., Tokyo, Japan). A C-to-T transversion at rs2290532, a C-to-T transversion at rs1902341, a G-to-T transversion at rs6779621 and an A-to-C transversion at rs11716090 were identified by the TaqMan-polymerase chain reaction method. Polymerase chain reaction was carried out using a GeneAmp polymerase chain reaction System 9700 thermal cycler (Applied Biosystems, Foster City, CA, USA). The fluorescence level of polymerase chain reaction products were measured using an ABI PRISM 7900HT Sequence Detector (Applied Biosystems), and this measurement was used to differentiate among the three genotypes.

\section{Statistical analysis}

Associations between the polymorphisms and clinical variables were analyzed using one-way analysis of variance. Differences in genotype or allele distribution were examined by $\chi^{2}$ analysis. Multiple regression analysis was used to assess the contribution of confounding factors. All numerical values are expressed as the mean \pm s.e.m. Statistical significance was defined as $P<0.05$. All statistical analyses were conducted using JMP software version 7 for Windows (SAS Institute, Cary, NC, USA).

\section{RESULTS}

\section{Construction of a linkage-disequilibrium block}

We attempted to construct a linkage-disequilibrium block at chromosome 3 p22.3 to define the region showing a strong association with $\mathrm{PAD}$ in our earlier genome-wide association study (in submission). The candidate SNPs (rs1902341, rs6779621 and rs11716090) belong to different tag SNPs based on HapMap information. We found that a coding SNP (cSNP), rs2290532 (D254N), existed in the neighboring sixth exon, very close to the three intronic SNPs (iSNPs), and it belongs to different tag SNP (Supplementary Figure 1a). As the possibility exists that the $\mathrm{D} 254 \mathrm{~N}$ amino-acid substitution in this cSNP could influence the overall protein function, we selected these four SNPs for further analysis.

\section{OSBPL10 polymorphism and lipid profile}

To elucidate associations between these SNPs and the risk factors for the metabolic syndrome, we examined these relationships in the Tanno and Sobetsu study population $(n=1188)$. Table 1 shows the basic characteristics of all study subjects. We performed genotyping of four SNPs (cSNP; rs2290532, iSNP; rs1902341, rs6779621, and
Table 1 Basic characteristics of study subjects $(n=1188)$

\begin{tabular}{lcc}
\hline & Male $(\mathrm{n}=455)$ & Female $(\mathrm{n}=733)$ \\
\hline Proportion (\%) & 38.3 & 61.7 \\
Age (years) & $66.5 \pm 0.5$ & $63.0 \pm 0.4$ \\
BMI (kg m${ }^{-2}$ ) & $23.9 \pm 0.2$ & $23.7 \pm 0.1$ \\
LDL Chol. (mg per 100 ml) & $117.2 \pm 1.3$ & $128.1 \pm 1.0$ \\
HDL Chol. (mg per 100 ml) & $51.3 \pm 0.6$ & $58.2 \pm 0.5$ \\
TG (mg per 100 ml) & $119.3 \pm 3.0$ & $96.4 \pm 2.4$ \\
BS (mg per 100 ml) & $102.9 \pm 1.0$ & $96.3 \pm 0.8$ \\
HbAlc (\%) & $5.37 \pm 0.04$ & $5.24 \pm 0.03$ \\
SBP (mm Hg) & $139.0 \pm 1.1$ & $137.3 \pm 0.9$ \\
DBP (mm Hg) & $78.3 \pm 0.6$ & $76.5 \pm 0.5$ \\
\hline
\end{tabular}

Abbreviations: BMI, body mass index; BS, blood sugar; DBP, diastolic blood pressure; HDL Chol., high-density lipoprotein cholesterol; LDL Chol., low-density lipoprotein cholesterol; SBP, systolic blood pressure; TG, triglyceride. Values are expressed as the mean \pm s.e.m. or $\%$.

Table 2 Relation of rs2290532 of OSBPL10 with serum lipid profile and blood pressure

\begin{tabular}{|c|c|c|c|c|}
\hline Genotype & $C / C$ & $C / T$ & $T / T$ & P-value \\
\hline \multicolumn{5}{|l|}{ Analysis for serum lipid profile } \\
\hline$n=1089$ & 692 & 349 & 48 & \\
\hline Proportion (\%) & 63.5 & 32.0 & 4.4 & \\
\hline Sex (male \%) & 39.1 & 40.2 & 43.8 & 0.794 \\
\hline Age (years) & $63.8 \pm 0.4$ & $64.9 \pm 0.6$ & $63.4 \pm 1.6$ & 0.333 \\
\hline $\mathrm{BMI}\left(\mathrm{kg} \mathrm{m}^{-2}\right)$ & $23.6 \pm 0.1$ & $24.1 \pm 0.2$ & $24.2 \pm 0.5$ & $0.020 *$ \\
\hline LDL Chol. (mg per $100 \mathrm{ml}$ ) & $123.8 \pm 1.1$ & $125.1 \pm 1.5$ & $111.6 \pm 4.1$ & $0.009 *$ \\
\hline HDL Chol. (mg per $100 \mathrm{ml}$ ) & $55.4 \pm 0.5$ & $55.1 \pm 0.7$ & $57.4 \pm 1.9$ & 0.521 \\
\hline TG (mg per $100 \mathrm{ml}$ ) & $103.0 \pm 2.2$ & $103.2 \pm 3.1$ & $105.4 \pm 8.5$ & 0.964 \\
\hline \multicolumn{5}{|l|}{ Analysis for blood pressure } \\
\hline$n=816$ & 522 & 257 & 37 & \\
\hline Proportion (\%) & 64.0 & 31.5 & 4.5 & \\
\hline Sex (male \%) & 37.5 & 39.3 & 35.1 & 0.836 \\
\hline Age (years) & $61.5 \pm 0.5$ & $63.0 \pm 0.7$ & $62.3 \pm 1.9$ & 0.244 \\
\hline $\mathrm{SBP}(\mathrm{mm} \mathrm{Hg})$ & $131.2 \pm 1.0$ & $131.9 \pm 1.4$ & $134.2 \pm 3.7$ & 0.707 \\
\hline $\mathrm{DBP}(\mathrm{mm} \mathrm{Hg})$ & $74.3 \pm 0.6$ & $76.0 \pm 0.8$ & $75.8 \pm 2.1$ & 0.182 \\
\hline
\end{tabular}

Abbreviations: BMI, body mass index; DBP, diastolic blood pressure; HDL Chol., high-density lipoprotein cholesterol; LDL Chol., low-density lipoprotein cholesterol; $n$, number of subjects; SBP, systolic blood pressure; TG, triglyceride.

$P$-values were calculated for allele data. ${ }^{*} P<0.05$. Values are expressed as the mean \pm s.e.m. or $\%$.

rs11716090), and the resulting genotype frequencies were not significantly different from the Hardy-Weinberg predictions (data not shown). According to the analysis of these genotypes, there was a significant correlation between LDL cholesterol (analysis of variance $P=0.009$ ) and the cSNP, rs2290532 (D254N) (Table 2), but there were no significant relationships among LDL cholesterol and the three iSNPs (Supplementary Table 1-3). The major allele of this cSNP correlated with a high serum LDL cholesterol level (Table 2). However, there were no significant differences in other risk factors for metabolic syndrome, including systolic blood pressure, diastolic blood pressure, HDL cholesterol and triglycerides (Table 2). Further analysis in a dominant model showed that mean serum LDL cholesterol level for individuals with the CC and TC allele was $12.7 \mathrm{mg}$ per $100 \mathrm{ml}$ higher than that for individuals with the non-risk TT allele (Supplementary Figure 1b). No significant relationships were observed between genotypes and HDL cholesterol or triglycerides $(55.3$ vs. $57.4 \mathrm{mg}$ per $100 \mathrm{ml}, P=0.521,103.1$ vs. $105.4 \mathrm{mg}$ per $100 \mathrm{ml}, P=0.964$, 
respectively). The relationship between the CSNP and LDL cholesterol was only significant for female subjects (Supplementary Figure 1b; Supplementary Table 4). To further clarify whether this cSNP is an independent factor in determining LDL cholesterol, we performed multiple regression analysis with adjustment for dyslipidemiarelated factors. The genotypic risk for hyper-LDL cholesterolemia remained after adjustment for age, sex and BMI, demonstrating that this polymorphism is an independent predictor of elevated LDL (Table 3).

\section{rs2290532 (D254N) polymorphism of OSBPL10 and LDL cholesterol}

The samples from public medical examinations were divided into groups of patients with dyslipidemia or normal cholesterol. Comparison of the genotype frequencies for both groups indicate that the non-risk allele reduced the risk of hyper-LDL cholesterolemia significantly $(P=0.003)$, with an odds ratio of 0.35 (95\% confidence interval $=0.17-0.76)($ Table 4$)$.

\section{DISCUSSION}

This study shows an association between polymorphisms in the OSBPL10 gene and serum LDL cholesterol level in a Japanese population. Although we analyzed four SNPs in the OSBPL10 gene, only the cSNP had a significant association with hyper-LDL cholesterolemia (Table 2). There were no significant associations between LDL cholesterol level and the three iSNPs (Supplementary Table 1-3). As this was not a case-control study, the sample number $(n=1188)$ might be too low to detect a significant difference for the iSNPs. In our earlier study, we were the first to report that SNPs in the OSBPL10 gene associates susceptibility to PAD by genome-wide association study in the Japanese population. Although the risk factors for PAD are diabetes mellitus, hypertension, dyslipidemia, smoking and being male, we did not find any associations between the OSBPL10 polymorphism and these risk factors aside from LDL cholesterol. This

Table 3 Multiple regression analysis for LDL cholesterol

\begin{tabular}{lrrrrr}
\hline Term & Estimate & s.e. & $\mathrm{t}$ & $\mathrm{P}$ & $\beta$ \\
\hline Age & 0.22 & 0.08 & 2.9 & 0.035 & 0.087 \\
Sex & 11.30 & 1.74 & 6.5 & $<0.0001$ & 0.193 \\
BMI & 1.47 & 0.25 & 5.8 & $<0.0001$ & 0.170 \\
rs2290532 [TT-TC and CC] & -6.35 & 2.04 & -3.1 & 0.019 & -0.091 \\
\hline
\end{tabular}

Abbreviation: BMI, body mass index; LDL, low-density lipoprotein

Sex: males $=0$, females $=1$; rs2290532: $\mathrm{TT}=0$, TC and $C C=1$. result suggests that OSBPL10 may be associated with $\mathrm{PAD}$ as a result of its effect on dyslipidemia. Very recently, Perttila et al. ${ }^{10}$ reported that OSBPL10 regulates cellular lipid metabolism and is associated with high triglycerides in Finnish subjects. However, in our analysis, the candidate SNP (rs11716090; tag SNP for rs2168422) and other SNPs were not associated with elevated triglycerides. A large-scale case-control study is necessary to further analyze the association between dyslipidemia and the OSBPL10 polymorphism in Japanese populations.

The function of OSBPL10 is not known, but there are several reports linking the OSBP/OSBP-related proteins (ORPs) to cholesterol homeostasis. Lagace et al. ${ }^{14}$ showed that OSBP overexpression in $\mathrm{CHO}$ cells results in a $40-60 \%$ decrease in acyl-CoA: cholesterol acyltransferase activity and its mRNA, a 50\% elevation in the mRNA levels of several sterol regulated genes (LDL receptor, HMG-CoA reductase and HMG-CoA synthase), and an $80 \%$ elevation in cholesterol biosynthesis. The observed changes in mRNA levels suggest that OSBP affects transcriptional control of the sterol homeostatic machinery. Possible mechanisms for the effects of OSBP on cellularcholesterol homeostasis are still unclear. Recent work has implicated OSBP/ORPs in the direct control of lipid synthesis and lipid transport in cells. ${ }^{15}$ OSBP/ORPs have been implicated as direct transporters of sterols, whereas other studies suggest that OSBP/ORPs act as sterol sensors that in turn modulate cellular functions, including signal transduction, vesicular transport and lipid metabolism. For instance, one study suggested that ORP1L is required for the translocation of late endosomes to microtubule minus ends. ${ }^{16}$ Similar to other members of the OSBP family, OSBPL10 has an oxysterol-binding domain and a $\mathrm{PH}$ domain, which attaches to non-ER membranes through binding of phosphatidylinositol. ${ }^{8,17}$ The identified cSNP (amino-acid substitution D254N) is located in the hinge region between two domains. This region is required for attachment to some organelles. In other family members, this region reportedly contains phosphorylation sites. Thus, we speculate that this cSNP may have an important function in the regulation of this protein.

\section{Study limitation}

Our study has several limitations. First, we were only able to enroll a small number of Japanese subjects. Second, this study had a crosssectional experimental design. Although dyslipidemia occurs mostly in aged individuals, the genotype-phenotype relationship should also be analyzed in a longitudinal study that is able to account for temporal changes. Third, the mechanisms by which functional (enzymatic) activities of OSBPL10 are regulated by rs2290532 (D254N) are unclear. Further study is required to clarify the function of rs 2290532 (D254N).

Table 4 Relation of rs2290532 of OSBPL10 to dyslipidemia

\begin{tabular}{|c|c|c|c|c|c|c|c|c|c|c|}
\hline & \multicolumn{4}{|c|}{ Dyslipidemia } & \multicolumn{4}{|c|}{ Normal } & & \\
\hline & \multicolumn{3}{|c|}{ Genotype } & \multirow[b]{2}{*}{ TT frequency } & \multicolumn{3}{|c|}{ Genotype } & \multirow[b]{2}{*}{ TT frequency } & \multicolumn{2}{|c|}{ Test for allele frequency } \\
\hline & $C C+C T$ & $T T$ & Sum & & $C C+C T$ & $T T$ & Sum & & $\mathrm{P}$-value & Odds ratio $(95 \% \mathrm{Cl})$ \\
\hline Hyper-LDL cholesterolemia & 381 & 8 & 389 & 0.02 & 742 & 44 & 786 & 0.06 & 0.003 & $0.35(0.17-0.76)$ \\
\hline Low-HDL cholesterolemia & 164 & 6 & 170 & 0.04 & 965 & 46 & 1011 & 0.05 & 0.36 & $0.77(0.32-1.83)$ \\
\hline Hyper-triglyceridemia & 224 & 12 & 236 & 0.05 & 905 & 40 & 945 & 0.04 & 0.78 & $1.21(0.63-2.35)$ \\
\hline
\end{tabular}

Abbreviation: LDL, low-density lipoprotein.

Hyper-LDL cholesterolemia, LDL $\geqq 140 \mathrm{mg}$ per $100 \mathrm{ml}$ or taking cholesterol-lowering drugs; low-HDL cholesterolemia, HDL $<40 \mathrm{mg}$ per $100 \mathrm{ml}$ or taking cholesterol-lowering drugs; hypertriglyceridemia, TG $\geqq 150 \mathrm{mg}$ per $100 \mathrm{ml}$ or taking cholesterol-lowering drugs. 


\section{CONCLUSION}

In conclusion, rs2290532 (D254N) of OSBPL10 might be associated with hyper-LDL cholesterolemia in Japanese subjects independent of age, sex and BMI.

\section{CONFLICT OF INTEREST}

The authors declare no conflict of interest.

\section{ACKNOWLEDGEMENTS}

We express gratitude to Kazuko Iwasa and Eriko Nagata for their continuous support of our investigations. This work was partly supported by a Grantin-Aid from the Ministry of Education, Culture, Sports, Science and Technology of Japan (21390223) and research grants from the Takeda Science Foundation.

1 Levanon D, Hsieh CL, Francke U, Dawson PA, Ridgway ND, Brown MS, Goldstein JL. cDNA cloning of human oxysterol-binding protein and localization of the gene to human chromosome 11 and mouse chromosome 19. Genomics 1990; 7: 65-74.

2 Gill S, Chow R, Brown AJ. Sterol regulators of cholesterol homeostasis and beyond: the oxysterol hypothesis revisited and revised. Prog Lipid Res 2008; 47: 391-404.

3 Kandutsch AA, Chen HW, Heiniger HJ. Biological activity of some oxygenated sterols. Science 1978; 201: 498-501.

4 Brown MS, Ho YK, Goldstein JL. The low-density lipoprotein pathway in human fibroblasts: relation between cell surface receptor binding and endocytosis of lowdensity lipoprotein. Ann NY Acad Sci 1976; 275: 244-257.

5 Wang X, Sato R, Brown MS, Hua X, Goldstein JL. SREBP-1, a membrane-bound transcription factor released by sterol-regulated proteolysis. Cell 1994; 77: 53-62.

6 Svensson S, Ostberg T, Jacobsson M, Norstrom C, Stefansson K, Hallen D, Johansson IC, Zachrisson K, Ogg D, Jendeberg L. Crystal structure of the heterodimeric complex of LXRalpha and RXRbeta ligand-binding domains in a fully agonistic conformation. EMBO J 2003; 22: 4625-4633.
7 Chen W, Chen G, Head DL, Mangelsdorf DJ, Russell DW. Enzymatic reduction of oxysterols impairs LXR signaling in cultured cells and the livers of mice. Cell Metab 2007; 5: 73-79.

8 Olkkonen VM, Johansson M, Suchanek M, Yan D, Hynynen R, Ehnholm C, Jauhiainen $M$, Thiele C, Lehto M. The OSBP-related proteins (ORPs): global sterol sensors for co-ordination of cellular lipid metabolism, membrane trafficking and signalling processes? Biochem Soc Trans 2006; 34: 389-391.

9 Fairn GD, McMaster CR. Emerging roles of the oxysterol-binding protein family in metabolism, transport, and signaling. Cell Mol Life Sci 2008; 65: 228-236.

10 Perttila J, Merikanto K, Naukkarinen J, Surakka I, Martin NW, Tanhuanpaa K, Grimard V, Taskinen MR, Thiele C, Salomaa V, Jula A, Perola M, Virtanen I, Peltonen L, Olkkonen VM. OSBPL10, a novel candidate gene for high triglyceride trait in dyslipidemic Finnish subjects, regulates cellular lipid metabolism. J Mol Med 2009; 87: 825-835.

11 Ohnishi H, Saitoh S, Takagi S, Ohata J, Takeuchi H, Isobe T, Katoh N, Chiba Y, Fujiwara $\mathrm{T}$, Akasaka H, Shimamoto K. Incidence of insulin resistance in obese subjects in a rural Japanese population: the Tanno and Sobetsu study. Diabetes Obes Metab 2005; 7: 83-87.

12 Fujiwara T, Saitoh S, Takagi S, Ohnishi H, Ohata J, Takeuchi H, Isobe T, Chiba Y, Katoh $\mathrm{N}$, Akasaka H, Shimamoto K. Prevalence of asymptomatic arteriosclerosis obliterans and its relationship with risk factors in inhabitants of rural communities in Japan: Tanno-Sobetsu study. Atherosclerosis 2004; 177: 83-88.

13 Akasaka H, Katsuya T, Saitoh S, Sugimoto K, Fu Y, Takagi S, Ohnishi H, Rakugi H, Ura N, Shimamoto K, Ogihara T. Effects of angiotensin II type 1 receptor gene polymorphisms on insulin resistance in a Japanese general population: the Tanno-Sobetsu study. Hypertens Res 2006; 29: 961-967.

14 Lagace TA, Byers DM, Cook HW, Ridgway ND. Altered regulation of cholesterol and cholesteryl ester synthesis in Chinese-hamster ovary cells overexpressing the oxysterolbinding protein is dependent on the pleckstrin homology domain. Biochem J 1997; 326(Part 1): 205-213.

15 Yan D, Lehto M, Rasilainen L, Metso J, Ehnholm C, Yla-Herttuala S, Jauhiainen M, Olkkonen VM. Oxysterol binding protein induces upregulation of SREBP-1c and enhances hepatic lipogenesis. Arterioscler Thromb Vasc Biol 2007; 27: 1108-1114.

16 Johansson M, Rocha N, Zwart W, Jordens I, Janssen L, Kuijl C, Olkkonen VM, Neefjes J. Activation of endosomal dynein motors by stepwise assembly of Rab7-RILPp150Glued, ORP1L, and the receptor betalll spectrin. J Cell Biol 2007; 176: 459-471.

17 Loewen CJ, Roy A, Levine TP. A conserved ER targeting motif in three families of lipid binding proteins and in Opi1p binds VAP. EMBO J 2003; 22: 2025-2035.

Supplementary Information accompanies the paper on Hypertension Research website (http://www.nature.com/hr) 